\title{
Fast full-field micro-tomography at the Nanoscopium multitechnique nanoprobe beamline of Synchrotron Soleil
}

\author{
K. Medjoubi ${ }^{1}$, G. Baranton ${ }^{1}$ and A. Somogyi ${ }^{1}$ \\ 1. Synchrotron Soleil, L'Orme des Merisiers Saint-Aubin - BP 4891192 GIF-sur-YVETTE \\ CEDEX \\ * Corresponding author, Kadda.medjoubi@synchrotron-soleil.fr
}

A fast full-field micro-tomography set-up has been integrated to the FZP based nanoprobe station of the Nanoscopium beamline (Synchrotron Soleil, France). It offers the possibility to have a 3D morphological preview of millimetre sized samples with micrometre resolution in less than 2 minutes prior to scanning nano-probe experiments. This new analytical possibility is especially useful for identifying buried structures in opaque samples for multimodal scanning imaging and ptychography.

The Nanoscopium hard X-ray $(5-20 \mathrm{keV})$ nanoprobe beamline is dedicated to multi-technique X-ray imaging with continuous fast scanning possibilities [1]. The beamline develops and offers complementary 2D/3D imaging and spectroscopy methods, also in association to ptychography imaging techniques. These multi-technique imaging possibilities pave the way towards the quantitative study of the morphology, elemental composition and chemical speciation of the samples at hierarchical length-scales in the $30 \mathrm{~nm}-1 \mu \mathrm{m}$ resolution range.

By combining both diffractive and reflective optics in one beamline, both flux hungry and high degree of coherence applications can be exploited successfully at this multi-technique beamline. The FZP based nano-probe station of Nanoscopium is dedicated to experimental techniques where high degree of coherence is a must, such as ptychography and nano-beam scanning multimodal imaging including absorption, differential phase contrast, dark field and X-ray Fluorescence. The detailed description of these scanning techniques and the data collection and management scheme, namely the Flyscan architecture, can be found in [2]. As an example Figure 1.a shows the absorption image of a Siemens star (NTT Advanced Technology Co.) measured at $14 \mathrm{keV}$ with $300 \mathrm{~nm}$ pixel size and $5 \mathrm{~ms}$ dwell time per pixel. The more than 200 Gbytes of multimodal raw data are reduced, corrected and reconstructed by the in-house developed software, MMX-I [3].
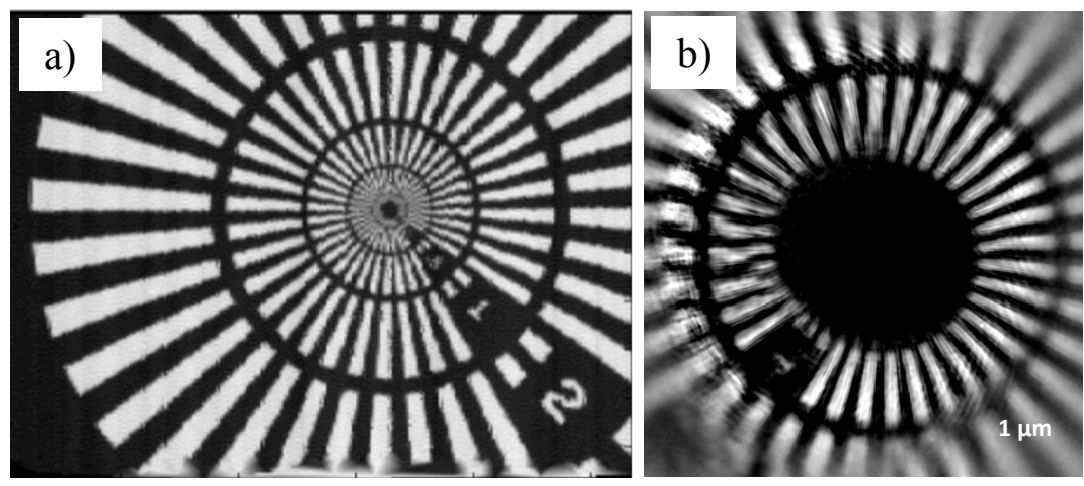

Figure 1 X-ray imaging of a Siemens star: a. Absorption

contrast, b. reconstructed Ptychography phase contrast

Ptychography [4] performed at $10 \mathrm{keV}$ in step scan mode in a region containing the finest features of $100 \mathrm{~nm}$ of the siemens star offers nanometer-scale resolution with quantitative phase contrast. The coherent diffraction pattern was recorded in the far field region by a 
photon-counting pixel detector, a quad Medipix 3RX with $55 \mu \mathrm{m}$ pixel size (Quantum Detectors 1td, UK). The reconstructed phase, by using the ePIE method by an in-house matlab code, is presented in the figure 1.b. The spatial resolution evaluated from the image by using the slanted edge method applied on a spoke edge was $35 \mathrm{~nm}$ (FWHM).

Present day scanning imaging techniques including X-ray ptychography are still relatively slow, and as a consequence 3D scanning tomography and large field of view high resolution ptychography is rarely performed due the necessary total measurement time of $>8$ hours. As such, these techniques are usually performed on a smaller region of the sample. In order to offer the possibility of identifying the region of interest for scanning X-ray techniques based on the complete 3D morphology of mm-sized samples we implemented fast (less than 2 minutes total measurement time) full field tomography with a $1 \mu \mathrm{m}$ resolution at the nanoprobe station. As such, a region of interest can be chosen in the very same experimental conditions for nano-meter resolution multimodal fast scanning 2D/3D imaging or Ptychography. The switch and alignment between the full field and scanning techniques is fast and straight-forward. The focusing optics are removed while a fast $2 \mathrm{D}$ imaging indirect detector with micrometer spatial resolution is inserted automatically into the beam. The data acquisition with continuous sample rotation and data collection are performed by using the Flyscan architecture.

As an example the full field and scanning XRF tomography of a $500 \mu \mathrm{m}$ large foraminifera, widely used as proxy in paleo-climatology and paleo-environment, have been measured. The volume rendering of the reconstructed fast full field tomography (Figure 2.a) allowed to identify a central region in the foraminifera (red rectangle in figure 2.a.) for fast multimodal scanning tomography. The acquired 3D data-set containing 2 millions pixels with micron resolution have been measured in 3 hours and resulted in a 500 GByte data file. Volume rendering of the dark field, phase contrast and the distributions of $\mathrm{Fe}$ and $\mathrm{Ca}$ are shown in figure 2.b. This example clearly illustrates the importance to have a preview of the sample morphology in order to select, without un-mounting the sample, a target zone for multimodal scanning imaging and/or ptychography.
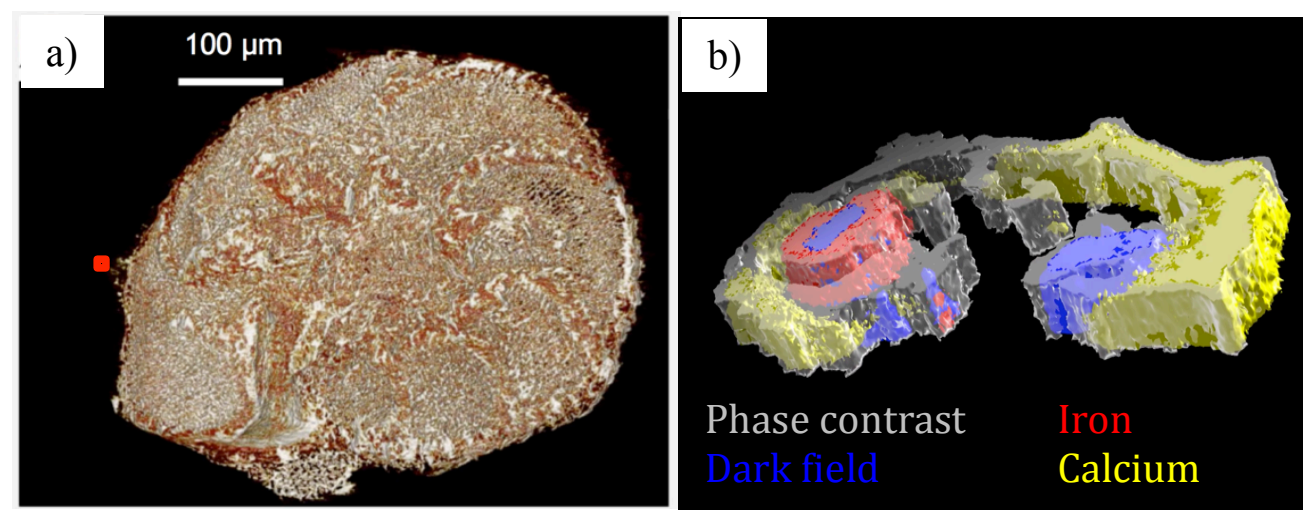

Figure 2 (a) Full field tomography and (b) Mutli-modal scanning tomography of a foraminifera

[1] A. Somogyi et al., (2015). Journal of synchrotron radiation, 22(4), 1118-1129.

[2] K. Medjoubi et al., (2013). Journal of synchrotron radiation, 20(2), 293-299.

[3] A. Bergamaschi et al., (2016). Journal of synchrotron radiation, 23(3), 783-794.

[4] F. Pfeiffer, (2018). Nature Photonics, 12(1), 9.

[6] The authors acknowledge N. Leclercq from the Soleil computing group for the Flyscan development and its integration to Nanoscopium and K. Desjardins from the Soleil detectors group for the indirect detector development. 